\title{
Effectiveness of personalized lifestyle intervention for community-dwelling, middle-aged and older patients with hypertension: evidence from a health promotion program in Chinese community
}

\author{
XiaoYue Yang ${ }^{1 \mathrm{a}}$, Ting Sun $^{2 \mathrm{~b}}$, Xiaoyu Zhang ${ }^{1}$, ZuChang Ma ${ }^{1 \mathrm{c}}$ \\ ${ }^{1}$ AnHui Province Key Laboratory of Medical Physics and Technology, Institute of Intelligent Machines, Hefei Institutes of Physical \\ Science \\ ${ }^{2}$ School of Nursing, Bengbu Medical College, Anhui, 233030, China
}

\begin{abstract}
The objective of this study was to preliminary evaluate the effectiveness of multicomponent, personalized lifestyle intervention for middle-aged and older patients with hypertension in a limitedresource Chinese community. A single-arm, pre-post intervention design was used. 169 communitydwelling patients were enrolled and trained community health workers conducted intervention activities in a local community health center. The intervention consisted of key strategies for promoting dietary habits, physical activity and health-related behaviors, where participants received individualized lifestyle guidance. Of enrolled participants, 122 completed the study. The 6-month intervention was efficient in improving dietary habits and health-related behavior. No significant changes was found in physical activity. Clinically significant were found in SBP by $8.3 \mathrm{mmHg}$, DBP by $4.1 \mathrm{mmHg}(\mathrm{p}<0.001)$ and improvements in secondary outcomes. Strategies for personalized intervention and motivational interviewing techniques provided great reference to the practice of public health care, with essential lessons learned from exercise promotion.
\end{abstract}

\section{Introduction}

An estimated 200 million adults in China have

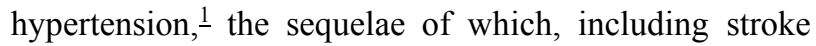
and heart diseases, are the leading causes of morbidity and mortality. ${ }^{2}$ The prevalence of hypertension is rapidly increasing, while the treatment and control rates are less than $50 \%$ and $20 \%$, respectively, with nearly two-thirds of hypertensives do not achieve adequate control of their blood pressure,, 3.4 which puts an enormous pressure on individuals, their families and public health care system.

It is well-established that behavioral and motivational strategies to help achieve healthy lifestyle are essential in the treatment of hypertension. . Substantial evidences on the effectiveness of community-based lifestyle intervention are well-documented in developed countries,,-7 which have significant public health implications for community hypertension management. However, these implications were mainly based on studies in high-income countries where patterns of lifestyle differ appreciably from those in developing countries, including China. ${ }^{8}$ Previous studies in China basically focused on the association of lifestyle factors with blood pressure control. .910 However, the effect of lifestyle intervention in a representing group of community-dwelling residents is not well described. A recent study on lifestyle modification for hypertensives in southern China underscored the need for more studies to add general evidence for primary health care. 11

In urban China, community health centers (CHCs) are major primary care providers. The Chinese health reform emphasized that $\mathrm{CHCs}$ should be served as the "health-gatekeeper" for hypertension management. $\frac{12}{}$ For lifestyle modification, 'one size fits all' intervention has been developed by general practitioners and community health workers (CHWs), in which the number of hypertensives engaged in actual behavior change is low. $\cdot$-13-15 Other studies have adopted lifestyle modification in community level,,$\underline{16,17}$ but few emphasized personalized and multicomponent approaches. Thus, we conducted personalized, comprehensive lifestyle intervention in an average level, resource-limited $\mathrm{CHC}$ for middle-aged and older patients with hypertension.

The aim of our program was to preliminary evaluate the effectiveness of the community-based lifestyle intervention with multicomponent and personalized intervention strategies, and to discuss the associated challenges, learned lessons, as well as potential solutions. More specifically, to test the efficiency of lifestyle promotion, including dietary, physical activity and health-related behavior, and investigate changes in blood pressure and secondary health outcomes. 


\section{Methods}

\subsection{Study setting}

This community-based health promotion program involved a collaboration between the local community healthcare committee and our study team. Most study activities, including screening, recruitment and lifestyle intervention, were carried out in the CHC. Study team provided standard training for CHWs, intervention support and materials. CHWs enrolled in the study received mandatory training and regular assessment. All aspects of the study were approved by the Ethics Committee of Hefei Institutes of Physical Sciences, Chinese Academy of Sciences (approval No. Y-2018-29). Informed consent was obtained from all individual participants included in the study.

\subsection{Participants and Recruitment}

The recruitment and screening interview took place in the local community located in Eastern China. The community healthcare committee provided a list of potential candidates, who deemed eligible for the study, and 347 patients were included. The study group used a purposive sampling method to select 234 of them. CHWs established contacts with candidates via telephone to invite them to participate, of whom 169 were enrolled in the study. A personal screening interview was carried out to obtain demographic information (Table 2) and written informed consent. Eligibility criteria were: (1) aged 50 to 79 years; (2) diagnosed with hypertension (registered as hypertensive patient in electronic health record system); (3) without severe organ damage, disability, cognitive impairment and other lifethreatening disease; (4) had lived in the community for at least 2 years; (5) able to provide written informed consent to participate in the study. Exclusion criteria were: (1) severe loss of vision, hearing, or communicative ability; disorders preventing participation in the study; (3) unable to participate in the follow-up visits.

\subsection{Intervention design}

During the 6-month intervention, CHWs conducted tailored, personalized intervention to each participant, following the standard procedure in the $\mathrm{CHC}$ and the study team supervised the whole process. The intervention consisted of two sessions, each lasted 3 month and was composed of one intervention meeting and two telephone follow-ups.

The first intervention meeting contained 4 components and the key strategies were summarized in Table 1 .

Table 1. Contents and key strategies for the intervention meeting

\begin{tabular}{|c|c|c|}
\hline Components & Contents & Key strategies \\
\hline Component 1 & Interviewing for lifestyle habits & $\begin{array}{ll}\text { - } & \text { Conducting participant-centered interviews } \\
\text { - } & \text { Using standard lifestyle questionnaire }\end{array}$ \\
\hline Component 2 & Health risk assessment & $\begin{array}{l}\text { - Applying the China-PAR risk prediction } \\
\text { model } \\
\text { - } \quad \text { Listing lifestyle-related risk factors }\end{array}$ \\
\hline Component 3 & Personalized lifestyle advice & $\begin{array}{ll}\text { - } & \text { Setting goals for the intervention } \\
\text { - } & \text { Offering personalized dietary advices } \\
\text { - } & \text { recommended intakes of nutrients } \\
\text { - } & \text { tips to measure food portions } \\
\text { - } & \text { Offering personalized exercise prescription }\end{array}$ \\
\hline
\end{tabular}

Component 4 Motivational interviewing(MI) techniques
- Integrating MI techniques into the meeting

- $\quad$ creating friendly atmosphere

- $\quad$ raising awareness

- discussing goals and beneficial effect

- $\quad$ providing detailed recommendations 


\subsubsection{Interviewing for lifestyle habits}

To have a comprehensive view of individual lifestyle preferences, CHWs conducted participant-centered interviews to collect information of lifestyle-related components concerning diet pattern, physical activity and behavioral habits regarding smoking and drinking by a standard questionnaire. The questionnaire was designed by the study team based on guidelines and a literature search for confirmed lifestyle-related questions $\frac{18-20}{}$ and was adapted by incorporating Chinese culture and tradition.

\subsubsection{Health risk assessment}

By applying the China-PAR risk prediction model, a validated tool with good ability to predict 10-year atherosclerotic cardiovascular disease (ASCVD) risk among Chinese population, 21 the risk of ASCVD was estimated for participants. The aim was to provide participants opportunities to be aware of their cardiovascular risk conditions and to attach importance to preventing cardiovascular diseases by controlling blood pressure level. The lifestyle-related risk factors were pointed out in proper order to encourage adopting healthier lifestyle practices for lowering ASCVD risk.

\subsubsection{Personalized lifestyle advices}

Individually-tailored lifestyle advices, based on lifestyle habits or preferences and current health status were given to each participant. The advices were divided into three parts: 1) goals for the intervention, classified as long-and short-term objectives for health promotion; 2) personalized and detailed advices for dietary modification. For instance, recommended intakes of different types of foods and nutrients which were in line with guidelines 19,20 and available meal plans that conformed to Chinese dietary pattern. In addition, tips to measure food portions were introduced in the form of pictures to help participants learn simple skills to estimate portion sizes in daily diet without a scale; 3) personalized exercise prescription for assisting participation in aerobic, resistance and stretching exercise. The prescription was made in consideration of current level of physical activity and exercise habits, based on ACSM's guidelines, 19 which consisted of recommended exercise program, time and frequency and the points for attention. Furthermore, the exercise program was graphically demonstrated.

\subsubsection{Motivational interviewing techniques.}

Motivational interviewing (MI) techniques were integrated into the intervention meeting, combined with lifestyle habits interviews, health risks assessment and lifestyle guidance. CHWs applied MI approaches previously used successfully in primary health care, 22,23 to support, encourage and enhance dietary, exercise and health-related behavior changes. During the meeting,
CHWs created a friendly atmosphere to build a solid foundation of trust with participants and the importance of individual ability to regulate behavior were emphasized. Furthermore, awareness of consequences of the behavior was raised by estimating the risk of ASCVD. Importantly, CHWs discussed goals and beneficial effect of the intervention, and explained detailed advices for promoting lifestyle to provide participants fundamental skills needed to achieve the goals.

After the first intervention meeting, participants would receive two regular follow-ups via telephone calls within the first session. CHWs reviewed the important points of the lifestyle modification and made sufficient communication to strengthen confidence for change.

At the end of first session, participants were asked to attend the second intervention meeting in CHC. During the second meeting, CHWs asked the participants about their recent lifestyle habits and whether the recommendations were implemented. When the changes did not happen, the barriers were discussed and CHWs addressed potential solutions for participants. Participants who promoted their habits were encouraged to keep the changes and were given further suggestions to achieve the long-term goals, including reinforced meal plans and exercise prescriptions.

\subsection{Measurements}

At the time of recruitment, demographic, educational information were acquired from electronic health record. Measurements of anthropometric and biochemical variables were collected by CHWs in CHC pre and post intervention. Body weight and height were measured by a digital scale, with participants wearing light indoor clothing and no shoes. Body mass index (BMI) was calculated as kilograms per squared meters $(\mathrm{kg} / \mathrm{m} 2)$. Waist circumference was measured with a horizontal tape measurement from the top of the right iliac crests. Hip circumference was measured at the widest circumference at the level of the symphysis pubis and gluteus maximus. After a 5-minute rest, in the supine position, blood pressure measurement, arterial stiffness measurement would be completed. Systolic blood pressure (SBP), diastolic blood pressure (DBP), pulse pressure (PP), central aortic blood pressure (CAP), augmentation index (AIx) were measured by IIM-2010A device (Institute of Intelligent Machines, Hefei, China). Brachial-ankle pulse wave velocity (baPWV) was derived by IIM-AS-100 device (Institute of Intelligent Machines, Hefei, China).

Lifestyle-related variables would be assessed through the above-mentioned questionnaire at the first intervention meeting and the end of intervention. The questions of dietary habits included the daily intake of cooking oil, salt, whole-grain, vegetable, water and milk. The standards for excessive or inadequate food intakes were as defined follows: high salt intake (more than $6 \mathrm{~g}$ salt a day), high fat intake (more than $30 \mathrm{~g}$ cooking oil a day), inadequate whole-grain intake (less than $250 \mathrm{~g}$ a day), inadequate vegetable intake (less than $300 \mathrm{~g}$ a day), 
inadequate water intake (less than $1500 \mathrm{ml}$ a day), inadequate milk intake (less than $300 \mathrm{~g}$ a day).20 Questions with respect to physical activity and exercise were consistent with International Physical Activity Questionnaire-Short Form (IPAQ-SF)18 and physical activity level was assessed based on guidelines.24 Regular exerciser was defined as at least 6 times per month for a mean duration of at least 30 minutes with a mean intensity corresponding to at least vigorous walking to jogging.25 For health-related behavior, smoking was defined as had smoked for more than 6 month and passive smoking as self-reported frequent exposure to secondhand smoke very often. Alcohol drinker referred to individual who claimed drank alcohol at least once per week and had drunk for more than 6 months.

\subsection{Statistical analysis}

All analyses were done using the IBM Statistical Package for the Social Sciences (SPSS) version 20.0 (SPSS Inc., Chicago, IL, USA). Descriptive statistics were presented as mean and standard deviation (SD) for continuous variables and percentage for categorical variables. Pre-intervention and post-intervention differences in categorical variables were tested by Chisquare test and continuous variables with paired test. A two-sided p-value $<0.05$ was considered statistically significant. Participants who withdrew or did not complete follow-ups were excluded in the analysis.

\section{Results}

\subsection{Baseline characteristics}

Of the 169 hypertensive patients enrolled in the study, 146 completed first intervention meeting. A total of 122 participants completed all intervention sessions and were included in the final analyses (Figure 1). The mean (SD) age of participants was $67.3(6.3)$ years, $59.8 \%$ were female; $56.6 \%$ had a high school education or above, $84.4 \%$ were unemployed or retired and $51.6 \%$ had family history of hypertension (Table 2).

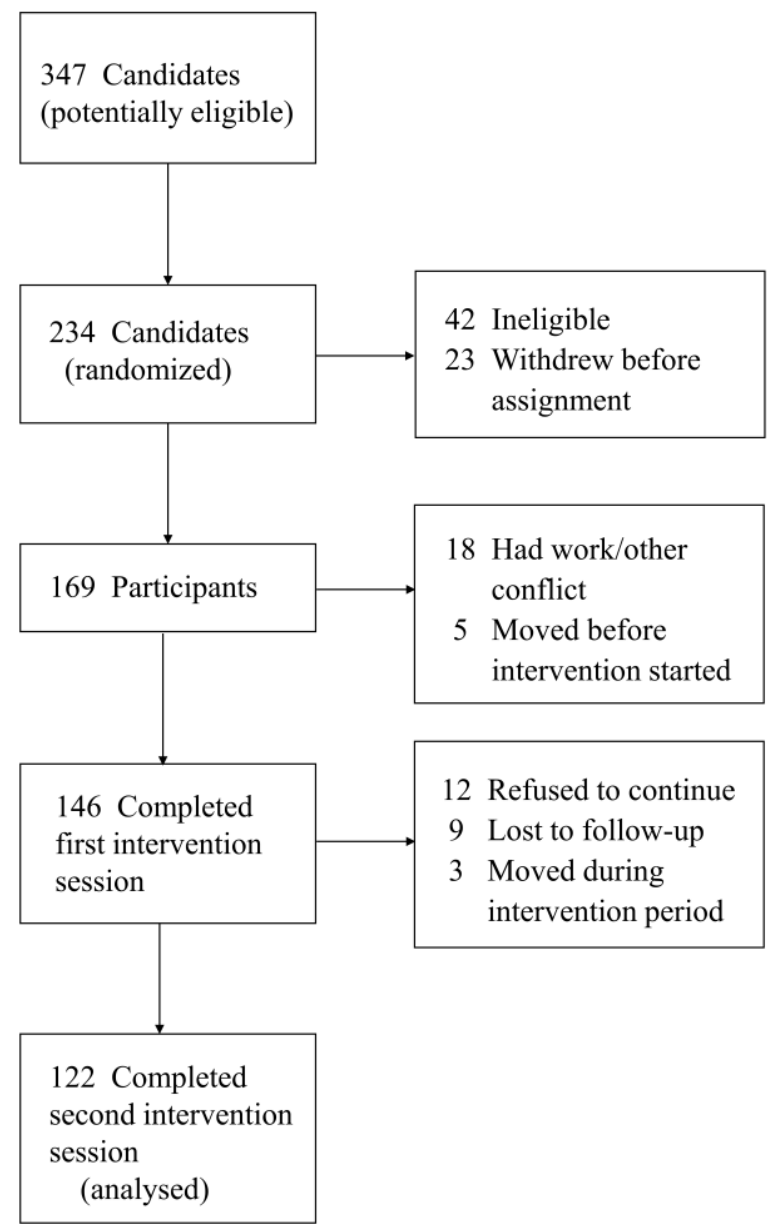

Figure 1. Study participants flow chart

Table 2. Demographic characteristics of the participants $(n=122)$

\begin{tabular}{lc}
\hline Variables & Mean (SD) or \\
\hline Age(years) & $67.3(6.3)$ \\
Gender & \\
Male & $49(40.2)$ \\
Female & $73(59.8)$ \\
Education level & \\
Primary school or lower & $53(43.4)$ \\
High school & $55(45.1)$ \\
College or above & $14(11.5)$ \\
Employment status & \\
Working & $19(15.6)$ \\
Unemployed or retired & $103(84.4)$ \\
Family history of hypertension & $63(51.6)$ \\
\hline Data were expressed as means (SD), or numbers with \\
percentages
\end{tabular}




\subsection{Changes in health-related lifestyle}

Health-related lifestyle pre and post intervention were given in Table 3 . There were significant positive shifts in the number of participants in high salt intake (decreased by $24.6 \%, \mathrm{p}<0.001$ ) and high fat intake (decreased by $21.7 \%, \mathrm{p}<0.001)$ after the intervention. Significant decrease in the number of participants in inadequate water intake (decreased by $12.3 \%, \mathrm{p}=0.045$ ) was also demonstrated. There were post-intervention reductions in numbers of participants in inadequate milk intake (decreased by $9.8 \%, \mathrm{p}=0.116$ ), whole-grain intake (decreased by $4.1 \%, p=0.517$ ), vegetable intake (decreased by $2.5 \%, \mathrm{p}=0.493$ ), though changes were not significant.

Table 3. Changes in lifestyle habits pre and post intervention $(\mathrm{n}=122)$

\begin{tabular}{|c|c|c|c|}
\hline Characteristics & Pre & Post & P-value \\
\hline \multicolumn{4}{|l|}{ Dietary habit } \\
\hline High salt intake & $48(39.3)$ & $18(14.8)$ & $<0.001$ \\
\hline High fat intake & $38(31.5)$ & $12(9.8)$ & $<0.001$ \\
\hline Inadequate whole- & $73(59.8)$ & $68(55.7)$ & 0.517 \\
\hline Inadequate & $12(9.8)$ & $9(7.4)$ & 0.493 \\
\hline Inadequate water & $51(41.8)$ & $36(29.5)$ & 0.045 \\
\hline Inadequate milk & $54(44.3)$ & $42(34.4)$ & 0.116 \\
\hline \multicolumn{4}{|l|}{ Physical activity } \\
\hline Low & $39(32.0)$ & $34(27.9)$ & \\
\hline Moderate & $60(49.2)$ & $62(50.8)$ & 0.756 \\
\hline High & 23(18.9) & $26(21.3)$ & \\
\hline $\begin{array}{l}\text { Regular exerciser } \\
\text { Health-related }\end{array}$ & $38(31.1)$ & $43(35.2)$ & 0.293 \\
\hline Smol & $21(17$ & $15(12.3)$ & 0.279 \\
\hline noking & $18(14.8)$ & $12(9.8)$ & 0.242 \\
\hline Alcohol drinking & $36(29.5)$ & $33(27.1)$ & 0.670 \\
\hline \multicolumn{4}{|c|}{$\begin{array}{l}\text { ata were expressed as numbers, with percentages. } \\
\text { Levels of participation in physical activity, based on } \\
\text { elf-report physical activity habits, pre and post } \\
\text { tervention were showed. At baseline, } 32.0 \% \\
\text { articipants had low physical activity level and only } \\
1.1 \% \text { kept regular exercise habits. Following the } \\
\text { tervention, the proportion of participants engaged in } \\
\text { ifferent levels of physical activity and number of } \\
\text { articipants maintained regular exercise habit were } \\
\text { ainly unchanged. }\end{array}$} \\
\hline
\end{tabular}

Mean changes in anthropometric and biochemical variables pre and post intervention were reported in
Table 4. Substantial differences were found in blood pressure, SBP decreased significantly by $8.3 \mathrm{~mm} \mathrm{Hg}$ $(\mathrm{p}<0.001)$, DBP by $4.1 \mathrm{~mm} \mathrm{Hg}(\mathrm{p}<0.001)$, PP by $3.1 \mathrm{~mm}$ $\mathrm{Hg}(\mathrm{p}<0.001)$ and CAP by $8.5 \mathrm{~mm} \mathrm{Hg}(\mathrm{p}<0.001)$. Anthropometric outcome measurements with significant improvements included weight, BMI and hip circumference.

Of secondary biochemical outcomes, the mean value of baPWV and AIx showed a trend of decreasing, although there was no statistical difference.

Table 4. Changes in anthropometric and biochemical variables pre and post intervention $(\mathrm{n}=122)$

\begin{tabular}{|c|c|c|c|c|}
\hline Variables & Pre & Post & $\mathbf{t}$ & P- \\
\hline \multicolumn{5}{|c|}{ Anthropometric Variables } \\
\hline Weight $(\mathrm{Kg})$ & $66.1(9.9)$ & $65.0(10$ & 3.91 & $<0.00$ \\
\hline BMI $\left(\mathrm{kg} / \mathrm{m}^{2}\right)$ & $25.8(3.2)$ & $\hat{25.4}(3.1)$ & 3.80 & $<0.00$ \\
\hline $\mathrm{WC}(\mathrm{cm})$ & $89.7(9.0)$ & 89.0 & 1.07 & 0.29 \\
\hline $\mathrm{HC}(\mathrm{cm})$ & $100.1(6.8)$ & $98.7 ̄(7.5)$ & 2.28 & 0.027 \\
\hline \multicolumn{5}{|c|}{ Blood pressure(mm Hg) } \\
\hline SBP & $131.8(14.7$ & $123.5(12$ & 5.87 & $<0.00$ \\
\hline DBP & $75.4(9.5)$ & $\hat{71.3(7.8)}$ & 5.52 & $<0.00$ \\
\hline PP & $56.4(12.6)$ & $52.3(11$ & 4.26 & $<0.00$ \\
\hline CAP & $123.8(15.7$ & $\widehat{11} 15.3(13$ & 5.25 & $<0.00$ \\
\hline \multicolumn{5}{|c|}{ Arterial stiffness index } \\
\hline AIx & $0.85(0.09)$ & $0.83(0.1$ & 1.82 & 0.071 \\
\hline baPWV & $16.57(5.28$ & $16.38(3$. & 1.16 & 0.247 \\
\hline
\end{tabular}

Data were expressed as means (SD).

Abbreviations: BMI, body mass index; WC, waist circumference; HC, hip circumference; SBP, systolic blood pressure; DBP, diastolic blood pressure; PP, pulse pressure; CAP, central aortic blood pressure; AIx, augmentation index; baPWV, brachial-ankle pulse wave velocity.

\section{Discussion}

In this study, a preliminary program was conducted to develop a comprehensive, personalized lifestyle intervention in $\mathrm{CHC}$ among middle-aged and older patients with hypertension in a limited-resource community. To our knowledge, this was the first study emphasized the multicomponent and personalized intervention strategies in a community level. One of main purposes was to investigate whether improvements in lifestyle could be achieved following the intervention and summarize learned lessons to provide evidence for primary health care. Differential effects were found in dietary habits, physical activity and health-related behavior (Figure 2). Encouraging results were achieved in dietary habits and health-related behavior, with insignificant changes in physical activity level and exercise habit. Another purpose was to investigate if intervention had a positive change on blood pressure. Significant reduction were demonstrated in SBP by 8.3 $\mathrm{mmHg}$, DBP by $4.1 \mathrm{mmHg}$, showing a marked effect on lowering blood pressure. 


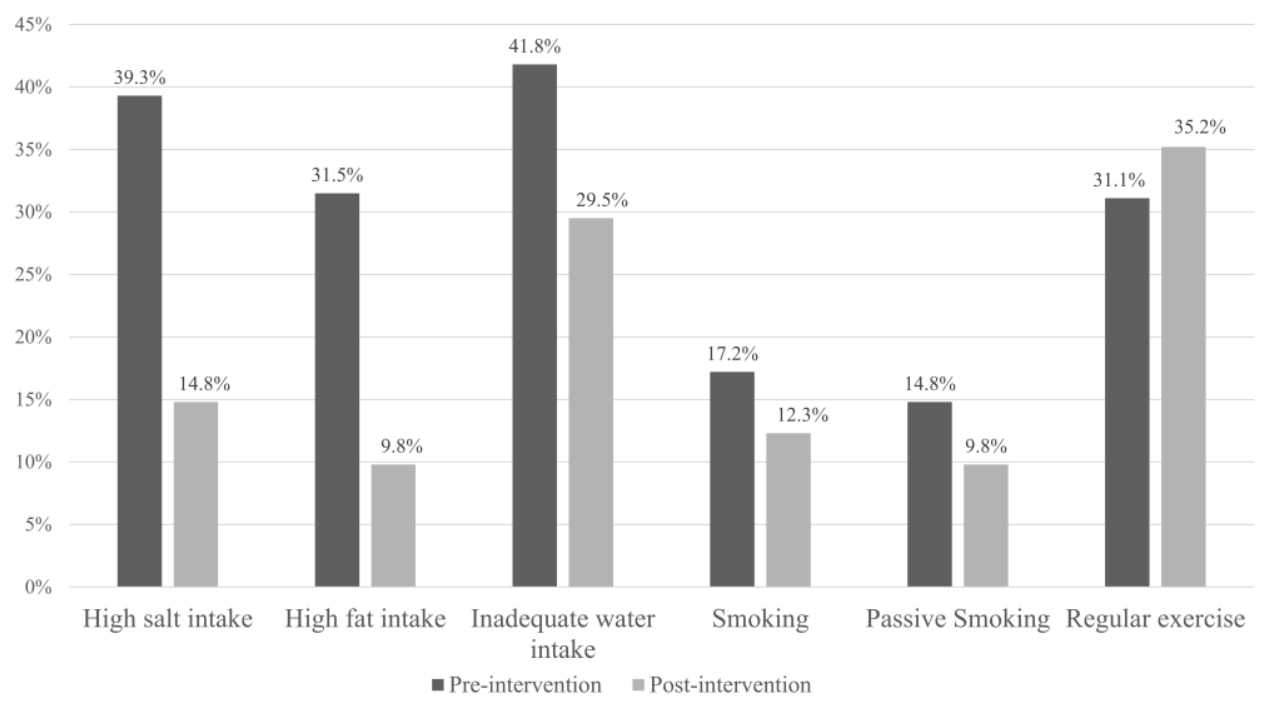

Figure 2. Lifestyle changes

The intervention responded favorably to dietary promotion, suggested the intervention could impact great effectiveness on dietary modification. Significant reductions in salt and fat intake were found, which also led to positive consequence in blood pressure. Low salt and fat dietary pattern is beneficial in blood pressure control, however, a large number of Chinese elderly have been accustomed to higher salt and fat dietary pattern. $\underline{26}$ In this study, tailored nutritional advices based on guidelines were offered, with particular consideration given to personal dietary habits. Therefore, the advices were accessible, available and acceptable to participants and improved their adherence to dietary change. Additionally, skills of estimating food portion was offered, with the purpose of acquiring simple and easy ways to scale the amount of food intake. Findings in dietary intervention were largely consistent with previous studies, $, 27,28$ led us to believe that personalized dietary advice should be considered an important tool in dietary intervention in public health.

An obvious trend toward improvement in healthrelated behavior was also observed. Percentage of participants engaged in smoking and drinking was dropping, the results showed no significance and it seemed reasonable to argue that this was due to the relatively small proportion of current smokers and drinkers at baseline.

These encouraging changes also suggested incorporating MI techniques in lifestyle intervention was essential, which motivated patients making healthy lifestyle choices by helping them exploring ambivalence, resistance and enhancing readiness to change. This finding was congruent with an earlier study among Chinese hypertensive patients, $\underline{\underline{29}}$ in which the patients in the MI group appeared to adhere to a healthy lifestyle better than those of the control group. In our study, MI techniques were integrated into several aspects of intervention meeting, including creating friendly atmosphere to realize participant-centered counseling, raising awareness by estimating cardiovascular health risk condition and providing detailed recommendations to change. Our study strengthened the need for MI techniques in primary health care.

Promotion in dietary and health-related behaviors resulted in significant reduction in blood pressure. In a similar study in Latino adults, $\frac{30}{}$ SBP decreased an average of $10.4 \mathrm{mmHg}$ after the six weekly group intervention sessions. Generally, a reduction of SBP by $5 \mathrm{mmHg}$ or of DBP by $2 \mathrm{mmHg}$ is considered as clinically significant. $\frac{31}{}$ In our study, the mean reduction of SBP was $8.3 \mathrm{mmHg}$, DBP as $4.1 \mathrm{~mm} \mathrm{Hg}$ and CAP as $8.5 \mathrm{~mm} \mathrm{Hg}$. Thus, our results revealed that personalized lifestyle intervention showed a clinically significant effect on reduction of SBP, DBP and CAP. For secondary outcomes, significant improvement was found in BMI. Indicators of arterial stiffness, AIx and baPWV both showed a slight increase although not significant. The result was plausible because the mean age of participants was 67 years and it was difficult to observe obvious change in arterial stiffness in a short time of 6 month.

No significant improvement was achieved in physical activity level and exercise habit. Of note is that changing routines of physical activity and exercise has been proved to be much difficult for elderly individuals. $\underline{32}$ Other studies showed successful promotion in physical activity among communitydwelling middle-aged and older adults. However, in most of those cases, intensive and supervised intervention concentrated on close contacts with participants were conducted in center-based settings, $\underline{33}$ which was difficult to carry out due to lack of resource in our community and was also hard to implement widely in general community settings. In our study, 
$32.0 \%$ participants were in low physical activity level and only $31.1 \%$ of participants had regular exercise habits at baseline, therefore personalized exercise prescriptions were designed for participants to encourage leisure-time physical activity. Exercise prescription included available exercise programs with no environmental restrictions, such as Tai chi, with recommended time and frequency. However, physical activity level and exercise habit did not show a statistically significant difference. This may be related to the lack of strict supervision and monitoring, using wearable activity monitors for instance. Moreover, a previous study to promote exercise on prescription in a Danish community, reported a higher motivation when participants engaged in a group training. $\frac{34}{3}$ Thus, for future study, in addition to exercise prescription for individual, organizing community-wide physical activity campaign for group exercise training would be recommended.

\section{Limitations}

There were several limitations to the study. First, the study was short-term, aimed at a small sample size, and effects were measured as within-participants changes. Considering the aim of this pilot study was to gather preliminary evidence of lifestyle intervention, the design allowed us to add to the literature of available strategies in primary healthcare practice. For generalizing more common practice for public health, a larger study incorporated with further experimental design was needed. Second, due to the restrictions of the study environment and resources, supervised and intensive intervention strategies were not developed. Third, the measures of physical activity and exercise were selfreported, typically leading to overestimation of the amount and intensity. As a result, the true level of physical activity and exercise habit may not exactly correlate with that reported by the participants. Similar limitation applied to dietary assessment. Future studies are warranted to avoid these limitations.

\section{Conclusion}

In summary, this preliminary health promotion program demonstrated significant improvements in dietary habits, health-related behaviors, which led to remarkable change in blood pressure and secondary outcomes. The results from this pilot study are encouraging, but more studies are needed to propose efficient approaches for physical activity promotion in community level. We feel that lessons learned from this pilot study could be expected to add great evidence to primary hypertension management in China.

\section{Limitations}

\subsection{Acknowledgements}

The authors would like to thank all the participants in the study, as well as the staff from the local residential committees and Jianghe community health center for their dedicated effort in making this study possible.

\subsection{Ethical approval}

This study was approved by the Ethics Committee of Hefei Institutes of Physical Sciences, Chinese Academy of Sciences (number: Y-2018-29). Informed consent was obtained from all individual participants included in the study.

\subsection{Funding}

This research was supported by Science and Technology Service Network Program of Chinese Academy of Sciences, China (grant KFJ-STS-ZDTP-033) and Major Special Science and Technology Project of Anhui Province of China (grant 18030801133). The funders had no involvement in the conduct of the research or preparation of the article.

\subsection{Conflict of interest}

The authors declare no conflict of interest.

\section{References}

1. Su M, Zhang QL, Bai XK, et al. Lancet. 390(10112):2559-2568. 2017

2. Zhou M, Wang $\mathrm{H}$, Zhu J, et al. Lancet. 387(10015):251-272. 2016

3. Lu JP, Lu Y, Wang XC, et al. Lancet.390(10112):2549-2558. 2017

4. Li Y, Yang L, Wang L, et al. International journal of cardiology. 227:516-523. 2017

5. Whelton PK, Carey RM, Aronow WS, et al. Hypertension (Dallas, Tex : 1979). 71(6):1269-1324. 2018

6. Puska P, Nissinen A, Tuomilehto J, et al. Annual review of public health. 6:147-193. 1985

7. Farquhar JW, Fortmann SP, Flora JA, et al. JAMA-J Am Med Assoc. 264(3):359-365. 1990

8. Yang GH, Wang Y, Zeng YX, et al. Lancet. 381(9882):1987-2015. 2013

9. Wang HHX, Wong MCS, Mok RY, et al. BMC Fam Pract. 16:10. 2015

10. Yin RX, Deng JQ, Yang DZ, et al. Kidney Blood Pressure Res. 29(5):312-320. 2006

11. Zhu XJ, Wong FKY, Wu CLH. Int J Nurs Stud. 77:171-178. 2018

12. Chen Z. Lancet.373(9672):1322-1324. 2009

13. Feng XL, Pang MF, Beard J. Bull World Health Organ. 92(1):29-41. 2014

14. Guo LJ, Bao Y, Ma J, et al. PLoS One. 13(5):17. 2018 
15. Huang W, Long H, Li J, et al. Global health research and policy. 3:18. 2018

16. Lu C-H, Tang S-T, Lei Y-X, et al. BMC public health. 15(1):33. 2015

17. Yu RJ, Yan LLJ, Wang HL, et al. Prev Chronic Dis. 11:7. 2014

18. Craig CL, Marshall AL, Sjostrom M, et al.Medicine and science in sports and exercise. 35(8):1381-1395. 2003

19. Medicine ACoS. ACSM's Guidelines for Exercise Testing and Prescription. Ninth edition ed: Lippincott Williams \& Wilkins; 2013.

20. Society TCN. The Food Guide Pagoda for Chinese Residents. http://dg.cnsoc.org/; . Accessed June20 2016.

21. Yang X, Li J, Hu D, et al. Circulation. 134(19):14301440. 2016

22. Brobeck E, Bergh H, Odencrants S, Hildingh C. J Clin Nurs. 20(23-24):3322-3330. 2011

23. Ogedegbe G, Chaplin W, Schoenthaler A, et al.American journal of hypertension. 21(10):11371143. 2008
24. Fan M, Lyu J, He P. Chinese Journal of Epidemiology. 35(8):961-964. 2014

25. Kujala UM, Kaprio J, Sarna S, Koskenvuo M. JAMA-J Am Med Assoc. 279(6):440-444. 1998

26. Shen X, Fang AP, He JJ, et al. Public Health Nutr. 20(16):2927-2936. 2017

27. Celis-Morales C, Livingstone KM, Marsaux CFM, et al. Int J Epidemiol. 46(2):578-588.2017

28. Armitage CJ, Conner M. Soc Sci Med. 52(10):15171524. 2001

29. Ma CH, Zhou Y, Zhou W, Huang CF. Patient Educ Couns. 95(2):231-237. 2014

30. Rocha-Goldberg MD, Corsino L, Batch B, et al. Ethn Health. 15(3):269-282. 2010

31. Verdecchia P, Gentile G, Angeli F, Mazzotta G, Mancia G, Reboldi G. Journal of hypertension. 28(7):1356-1365. 2010

32. Justine M, Azizan A, Hassan V, Salleh Z, Manaf H. Singapore medical journal. 54(10):581-586. 2013

33. Olanrewaju O, Kelly S, Cowan A, Brayne C, Lafortune L. PLoS One. 11(12):19. 2016

34. Roessler KK, Ibsen B. Journal of Public Health. 17(3):187-193. 2009 